\title{
Addendum: Observation of an anti-PT-symmetric exceptional point and energy-difference conserving dynamics in electrical circuit resonators
}

\author{
Youngsun Choi ${ }^{1}$, Choloong Hahn², Jae Woong Yoon ${ }^{1,3} \&$ Seok Ho Song ${ }^{1}$
}

Addendum to: Nature Communications https://doi.org/10.1038/s41467-018-04690-y, published online 05 June 2018.

In this Article, we describe that a PT-symmetric Hamiltonian $H^{(\mathrm{PT})}$ and anti-PT-symmetric Hamiltonian $H^{(\mathrm{APT})}$ are related by a similarity transformation with a unitary operator $U$ given in Eq. (7). This statement may cause confusion or incorrect arguments due to its mathematical validity limitation. Therefore, here we provide an additional explanation. This relation is strictly valid only if $H^{(\mathrm{PT})}$ and $H^{(\mathrm{APT})}$ are traceless and off-diagonal elements in $H^{(\mathrm{PT})}$ are purely real. In case of non-traceless cases, one can employ an energyshifting gauge transformation that renders $H^{(\mathrm{PT})}$ and $H^{(\mathrm{APT})}$ traceless, in order to make valid use of the relation. In addition, a similarity relation between traceless $H^{(\mathrm{PT})}$ and $H^{(\mathrm{APT})}$ can be extended for $H^{(\mathrm{PT})}$ with complex-valued off-diagonal elements by a generalized unitary-transformation operator

$$
U^{\prime}=\frac{1}{\sqrt{2}}\left[\begin{array}{ll}
1 & \mathrm{e}^{i \theta} \\
\mathrm{e}^{-i \theta} & -1
\end{array}\right],
$$

where $\theta$ is phase angle of an off-diagonal element of a traceless $H^{(\mathrm{PT})}$.

Published online: 30 May 2019

(C) Open Access This article is licensed under a Creative Commons Attribution 4.0 International License, which permits use, sharing, adaptation, distribution and reproduction in any medium or format, as long as you give appropriate credit to the original author(s) and the source, provide a link to the Creative Commons license, and indicate if changes were made. The images or other third party material in this article are included in the article's Creative Commons license, unless indicated otherwise in a credit line to the material. If material is not included in the article's Creative Commons license and your intended use is not permitted by statutory regulation or exceeds the permitted use, you will need to obtain permission directly from the copyright holder. To view a copy of this license, visit http://creativecommons.org/licenses/by/4.0/.

(c) The Author(s) 2019

\footnotetext{
${ }^{1}$ Department of Physics, Hanyang University, 222 Wangsimni-Ro, Seoul 04763, Korea. ${ }^{2}$ School of Electrical Engineering and Computer Science, University of Ottawa, 800 King Edward Avenue, Ottawa, ON K1N 6N5, Canada. ${ }^{3}$ Electronics and Telecommunications Research Institute, Daejeon 34129, Korea. These authors contributed equally: Youngsun Choi, Choloong Hahn, Jae Woong Yoon. Correspondence and requests for materials should be addressed to J.W.Y. (email: jaeong.yoon@gmail.com) or to S.H.S. (email: shsong@hanyang.ac.kr)
} 